\title{
Research and Exploration on Intelligent Substation Information Integration Applications
}

\author{
Chengming Jin \\ Information and Communication Branch State Grid \\ Liaoning Electric Power Supply Co. Ltd \\ Shenyang, China \\ jcm@ln.sgcc.com.cn \\ Yongbin Zhao \\ Information and Communication Branch State Grid \\ Liaoning Electric Power Supply Co. Ltd \\ Shenyang, China \\ zhaoyb@ln.sgcc.com.cn
}

\author{
Wei Li \\ Information and Communication Branch State Grid \\ Liaoning Electric Power Supply Co. Ltd \\ Shenyang, China \\ lw1@ln.sgcc.com.cn \\ Hai $\mathrm{Yu}$ \\ Jinzhou Power Supply Branch \\ State Grid Liaoning Electric Power Supply Co. Ltd \\ Shenyang, China \\ yuh@ln.sgcc.com.cn
}

\begin{abstract}
The rapid development of the market economy promote the development of China's power grid career in recent years, the smart grid construction has gradually become an important power grid construction in content, and received wide attention. In the process of building a smart grid, you need a strong flow of information, traffic and power flow as a basis. When the third achieve organic integration, it is able to meet the requirements of smart grid construction. In this case, the integration of intelligent substation information construction platform makes some information silos to be broken to achieve timely data collection and share substation in promoting the construction of smart grid plays an important role. On this basis, based on the intelligent substation IEC61850 standard modeling, information and communication technology has become the basis of information integration platform for intelligent substation construction. This article will mainly focus on issues related to the integration of intelligent substation information simple analysis.
\end{abstract}

Keywords- development;grid;flow; intelligent

\section{INTRODUCTION}

Intelligent Substation including intelligent high-voltage equipment and substations unified information platform in two parts. Intelligent high-voltage equipment including smart transformers, intelligent high-voltage switchgear, transformers and other electronic. Intelligent transformer and control system relies on communication fiber connected to grasp the transformer status parameters and operating data. When the operation mode is changed, the device according to the voltage, the power of the system, deciding whether to adjust the tap; When a problem occurs the device will provide early warning and status parameters, reduce operation and management costs to a certain extent, reduce risk, improve transformer reliability. Intelligent high-voltage switchgear and control equipment, switching with higher performance, equipped with electronic devices, sensors and actuators, with monitoring and diagnostic functions. Electronic transformer means pure fiber transformers, transformers and other magnetooptical glass, which can effectively overcome the shortcomings of conventional electromagnetic transformers. Substation unified information platform features are the two transverse information sharing systems, mainly for the management system of the upper application of various information obtained unification; the second is the standardization of longitudinal information system, mainly for the upper layers of their application support transparency.

As microelectronic technology, network communication technology and computer control technology continues to develop, intelligent automatic device microprocessor core grid control has been widely used; the same rate as the substation equipment reliability and availability requirements continues to improve and increase the voltage and current measurement technology, electronic transformer applications and IEC61850 standards promulgated, the idea evolved digital substation, power industry exhibition this extensive research practice: work through a number of engineering practice, China has been put into the dozens to IEC61850 communication standards, using station level, process level, the spacer layer three-tier structure, network sharing and sampling data Go OSE (generic object oriented substation event, generic Object Oriented substation Event) trip for sharp Features of digital substation[1]. However, by engineering practice found a secondary device interface issues between devices has not been solved, which led directly to achieve the status of 
maintenance and other functions will be restricted; equipment and station level database for each master function is not effective integration, although some have realized the intelligent features, but poor application effect; interactive information and scheduling end station terminals, and poor plant stand between the ends [2].

At present, the development of smart grid (SMART GRID) at the United States and Europe have risen to the national strategic level, an important part of national economic development and energy policy, the domestic smart grid has also done a lot of research work; as power saving substation, its automated system for data collection, analysis and upload, pretreatment of the accident, the implementation of a wide area, such as the behavior of the instruction has an irreplaceable role, so the degree of intelligence to some extent determines the smart grid of degree[3].

In recent years, with the benefit due to severe energy shortages, the development of low-carbon economy has become the consensus of the whole society. At the same time, users of the power supply reliability requirements continue to increase, the development of electric cars and other utilities for energy distribution and storage as well as new demands; in this case, the grid operator faces enormous challenges [4]. To address these challenges, the need to develop clean and efficient energy and diversified energy, the development of distributed energy and microgrid, to ensure energy security and grid stability, security and economic operation. In order to respond flexibly to access large-scale distributed energy connected to the grid and micro-grid coordinate systems to ensure clean power and efficient operation, the development of China's smart grid features slightly stronger future is to deal with the new situation facing the development of the grid is to build stable, objective needs of the economy, clean, safe, modern energy supply system; adapt to China's national conditions, to meet the needs of the future development of the strategic choice of all aspects, it is important for the harmonious development of China's economic and social protection.

Smart Grid is the inevitable trend of modern social and economic development, but also to ensure continuous power and the basic guarantee for safe operation. Currently, countries around the world have for the future development of the smart grid vision and framework put forward for China's power grid, said Ali, which itself has a unique operating characteristics, drawing on the experience of developed grid home construction based on the combination of technology and network development factors power requirements, to achieve power flow, and the integration of information flow and traffic flow will be the inevitable trend of China's smart grid development. Intelligent variable electrical smart grid construct as a node in the device, its major role in achieving the perfect worry about between three kinds of information to enable them to meet the needs of different types of grid operation client[5]. And to achieve this goal is bound to order the construction of integrated information platform for infrastructure, achieve substation modeling and running all the data in the platform, and the panorama of the station to conduct a comprehensive analysis of data and applications, which is the development of intelligent substation the inherent requirements.

\section{THE CONSTRUCTION OF INTELLIGENT SUBSTATION INFORMATION INTEGRATION PLATFORM}

Currently, the traditional information systems, including the construction of a substation in a variety of types, such as SCADA, fault information systems, etc., are arranged between the different systems in accordance with certain rules, but where there are some drawbacks, some systems information collection and processing prone to overlap and duplication, and in the lateral system also prone islands of information, the existence of such defects, resulting in a waste of system resources, but also cannot guarantee the completeness and accuracy of the data, and therefore makes use of substation information subject to certain restrictions. Advances in science and technology, promote the rapid development of information technology substation, at the same time, in order to meet the information needs of different users, resulting in a smart substation and the corresponding information acquisition and application mode.

\section{A . Intelligent Substation Information Integration Design}

In a conventional substation system, comprising a plurality of operating platforms and operating rules, the network structure is also more complex; when the information transfer is too large, it is prone to clogging or delays, lead to slower operating efficiency, and maintenance are also very late difficulties. But in the smart substations, IEC61850 standard system using object-oriented modeling techniques for on-site equipment can be directly used for data modeling object attribute operation, the traditional secondary device features integrated management, through a unified access At the same time external access interface, making the secondary system structure has been greatly simplified, system reliability has increased significantly, which has become the basis for intelligent substation information technology integration design[6].

Based on the standard IEC61850 substation intelligent information integration platform, the need to adopt a hierarchical distributed processing method, and follows the principles of decentralization, decentralization of functions of different network platform layer. In order to meet the requirements of the building a comprehensive platform for information transmission, need to follow the different levels of information processing required to select the priority level troubleshooting, and to obtain information on the different network layers were aggregated and analyzed in order to establish compliance with IEC61850 standard logic nodes meet extended data objects and application requirements.

\section{B. Intelligent Substation Information Integration Platform Architecture.}

Intelligent substation information of the architecture process on integration platform needs to use information and data from the substation outside the station [7]. After the data processing, real-time, secure data information for users inside and outside the station. Intelligent Substation system collects data mainly includes the following aspects: 
data grid operation, including the power grid is in a normal operating state when the voltage and current data; high-voltage substation equipment related data, including high-voltage substation equipment operation condition monitoring and related data reflect substation adjacent operating equipment condition monitoring data; substation located adjacent to the operating data, primarily to reflect the operational status of the adjacent substation monitoring data, the site between adjacent substation data associated with the state; reflect substation protection and control equipment, and data from other equipment operating status; relevant information to ensure the stable operation of the substation other data environments; user's data[8].

The process of building intelligent substation information integration platform, the need for different data and evaluate the reliability of the information separately, different data have different levels of needs in terms of reliability, so it needs to be through the construction of information platform different treatment, in order to ensure the validity of the information. This information integration platform is by a central unit and a plurality of sub-units, through different units for different hierarchical data information distributed processing. Each sub-unit has a fixed observation area, and data acquisition and processing within the region, and to develop different levels of information sources based on predetermined information extraction rules, and placed in different spacer layer, according to the different needs of users transferred to the user information. Under normal circumstances, the position of each sub-unit is in the position of the spacer layer for a device system, the different spacer layer to form a complete device by combining function. Functional sub-units is usually set according to different spaced positions, functions and information users and be separately provided, so that it can be different according to the sub-units do not have the processing power to effectively tie, thus forming a complete database and information library that provides the data foundation for the continued operation of intelligent substation[9]. In general, information integration platform configuration data center server unit, sub-unit substation each application should be interconnected via standard data bus and a data server interface with the central unit, to meet the real-time data and non-real-time data storage and requires read. Data server should have the ability to isolate the district, according to "secondary power system security protection overall program" and "substation secondary system security plan" for the different zones to isolate data storage.

\section{Intelligent Substation Information Integration Platform for Functional Design}

The construction of intelligent substation information integration platform, the need for intelligent substation internal data and information collection, and the need for timely treatment, complete identification data, estimates, and other aspects of integration, and ultimately to the substation intelligent management. According to current operational needs intelligent substation, the intelligent substation information integration platform should have the following functions:

(A) Operational monitoring. Operation monitoring function can be achieved on intelligent substation equipment operating status and full monitoring of relevant data, in order to ensure stable operation of the substation system. Through information integration platform to achieve timely access to monitoring data, and provide the basis for the normal operation of the system, through the station back to all of the data to be displayed [10].

(B) Operation and control functions. Operation and control functions mainly use for intelligent substation equipment operation and control, and the control of the main methods of remote central control station and control. Remote central control station is to use the data provided by the remote computer equipment, substation equipment running state judge, thereby issuing the appropriate command based on the judgment result, in order to achieve effective control of substation equipment. When the station control mainly perform daily operational status of the device to control abnormal operating conditions encountered, can take effective operational strategies to ensure the normal operation of the substation equipment.

(C) Information analysis and intelligent alarm. During the substation operation, the need for operating data under different operating conditions to collect, analyze and deal with simultaneously, ultimately through effective human-machine interface for output, providing more data reference for the operator. At the same time, the system can function as substation fault information based on certain prediction, while made alarm information, through the fault of the previous data analysis and comparison of the failure to make a timely diagnosis.

(D) Operation and management functions. This feature is mainly for real-time data and historical information for online monitoring of substation operation, while monitoring the implementation of state power transmission and achieve timely interaction with other data information, analyzed by the quality of information exchange on operational status of the device, thus for device management and early warning capabilities. At the same time, the system is able to achieve timely scheduling platform to achieve integrated management of substation map mode.

\section{THE AdVANTAGE OF INTELLIGENT SUBSTATION}

\section{A . Achieve good environmental effects of low-carbon}

Intelligent substation, the traditional cable connection is no longer applied engineering, replaced by fiber-optic cable, in various types of electronic equipment used in a large number of high integration and low power consumption of electronic components, in addition to the traditional oil-filled mutual also did not escape the fate of being eliminated, the electronic transformer to be replaced. Whether or improve wiring means of a variety of devices, are effective in reducing energy consumption and waste, not only reduces costs, but also effectively reduces the damage inside the substation electromagnetic radiation 
pollution on people and the environment formed in a very large extent, improve the quality of the environment, to achieve the optimal performance of the substation, so that the ability of environmental protection is more significant.

\section{B. Having good interaction}

Operating characteristics of intelligent substation responsibilities and burdens, it must have a good interaction. Statistical work which is responsible for the power grid, it asked him back to the grid must have safe, reliable, accurate and detailed information about the function. After the realization of smart substation collection and analysis of information, not only to share information internally, it can be more complex and intranet, make a good interaction between the advanced system. Smart grid interactive power grid to ensure the safety and stable operation.

\section{Reliability Features}

The customer is one of the basic requirements for electricity reliability, intelligent substation with a high degree of reliability, to meet the customers' needs, but also to achieve a high power operation. Because there is a system of substation, led by a launch body prone to the phenomenon, it is inside the substation itself and all facilities are highly reliable, such characteristics will require substation needs to have detection, fault management functions, only with this function can only be effective in preventing the emergence of the substation failure after failure and can be processed quickly, so that the working conditions of the substation remains in the best condition.

\section{CONCLUSION}

With the continuous development of science and technology, power utilities also get more space for development, at the same time, the scope of application of intelligent substation are increasingly widespread.
Intelligent substation technology will also go through a lengthy process of development, in terms of technology and programs need to be constantly strengthened and improved, it has a better adaptability and professionalism. Intelligent substation information technology will also be integrated with the development of intelligent substation and continuous improvement.

\section{ACKNOWLEDGMENT}

The authors gratefully acknowledge the contribution of co-workers and reviewers' comments.

\section{REFERENCES}

[1] S.X. Huang and T.G. Huang, "System reliability power protection devices running explore,” Nor.Elec.Pow.Tech, vol.7,pp.19-24, July 2011

[2] C.Xue and S.B. Li, " Reliability Theory inrelay protection," Ele.Pow.Auto.Equip, vol.7,pp.32-37, May 2011

[3] H.T.Jiang,Y.Yue and X.L.Yang.,"Protection coordination of distributed generation and distribution," Elec.Pow.Auto.Equip, vol.7,pp.86-90, July 2011

[4] H.P.Wang and W.Yang, "Reliability and protection of Dynamic Performance Simulation," Pow.Cons, vol.1,pp.15-19, April 2012

[5] A.L.Chen and H.D.Zhang, "Summary protection reliability study," Auto.Elec.Pow.Syst, vol.9,pp.23-28, June 2012

[6] R.Yu, "The development of online protection intelligent early warning system," Elec.Pow.Auto.Equip,vol.32,pp.33-36, May 2009

[7] B.H.Zhang, "Progress protection technology,"Pow.Cons, vol.24,pp.43-48, May 2012

[8] W.Y.Huang, " Review on Development of new protection," Ele.Pow.Auto. Equip, vol.7,pp.23-27, May 2011

[9] H.L.Zeng, "Substation operation Problems and Countermeasures," Tech.Wind, vol.13, pp.14-18, April 2012

[10] L.Zhou and R.L.Zan, "On the relay in the substation reliability applications running," Auto.Elec.Pow.Sys, vol.28, pp.48-53, April 2010 